\title{
Dispersion of Multidrug-Resistant Enterococcus faecium Isolates Belonging to Major Clonal Complexes in Different Portuguese Settings $\nabla$
}

\author{
Ana R. Freitas, ${ }^{1,2}$ Carla Novais, ${ }^{1,3}$ Patricia Ruiz-Garbajosa, ${ }^{2,4,5}$ \\ Teresa M. Coque, ${ }^{2,4,5} \dagger$ and Luísa Peixe ${ }^{1} \dagger^{*}$ \\ REQUIMTE, Laboratório de Microbiologia, Faculdade de Farmácia, Universidade do Porto, Porto, Portugal ${ }^{1}$; Servicio de \\ Microbiología, Hospital Universitario Ramón y Cajal, Madrid, Spain²; Faculdade Ciências da Saúde, Universidade \\ Fernando Pessoa, Porto, Portugal ${ }^{3}$; CIBER en Epidemiología y Salud Pública (CIBERESP), Madrid, \\ Spain ${ }^{4}$; and Unidad de Resistencia a Antibióticos y Virulencia Bacteriana Asociada al \\ Consejo Superior de Investigaciones Científicas (CSIC), Madrid, Spain ${ }^{5}$
}

Received 25 December 2008/Accepted 12 May 2009

\begin{abstract}
The population structure of 56 Enterococcus faecium isolates selected from a collection of enterococci from humans, animals, and the environment in Portugal (1997 to 2007) was analyzed by multilocus sequence typing. We identified 41 sequence types clustering into $\mathrm{CC17,} \mathrm{CC5,} \mathrm{CC9,} \mathrm{CC22} \mathrm{and} \mathrm{CC94,} \mathrm{all} \mathrm{clonal} \mathrm{lineages} \mathrm{com-}$ prising isolates from different hosts. Our findings highlight the role of community-associated hosts as reservoirs of enterococci able to cause human infections.
\end{abstract}

Enterococci are organisms widely distributed in nature that are recognized as one of the leading causes of nosocomial infections $(1,20)$. Despite their ubiquity, the population structure of Enterococcus faecium comprises a diversity of sequence types, with overrepresentation of particular clonal complexes (CCs) associated with swine (CC5), poultry (CC9), veal calf (CC1), or humans (CC17, CC22, and CC94) $(1,5,15,17,30$, 37). Particular host-specific human clonal lineages are considered high-risk CCs (HiRCCs) since they are recovered mostly from hospitalized patients (17). CC17 is the only recognized $E$. faecium HiRCC, nowadays globally disseminated, which has been sporadically isolated from nonhospitalized humans (3, 8-10). Different studies have analyzed the population structure of local enterococci, but they are focused mainly on clinical strains with a specific phenotype, such as vancomycin resistance, generally isolated in a short temporal frame $(2,7,16,18$, 27, 29, 36).

We analyzed 56 representative E. faecium isolates from a Portuguese collection comprising 1,700 enterococci from different geographical locations (1997 to 2007), some included in previous studies (22-26). They were recovered from hospitalized patients in five hospitals of different regions $(n=20)$, swine excrement $(n=6)$ and environmental piggery samples $(n=16)$, retail poultry $(n=6)$ of four national commercial brands, feces from healthy humans $(n=5)$, hospital wastewater $(n=2)$, and the estuary of the river Douro $(n=1)$. Susceptibility testing with 15 antibiotics was performed following CLSI guidelines (6). Species identification and detection of genes encoding vancomycin resistance were performed by us-

\footnotetext{
* Corresponding author. Mailing address: Laboratório de Microbiologia, Faculdade de Farmácia, Universidade do Porto, Rua Aníbal Cunha, 164, 4050-047 Porto, Portugal. Phone: 351-2-22078946. Fax: 351-2-22003977. E-mail: lpeixe@ff.up.pt.

$\dagger$ Luísa Peixe and Teresa M. Coque contributed equally to the direction, preparation, and writing of the manuscript.

${ }^{\nabla}$ Published ahead of print on 15 May 2009.
}

ing multiplex PCR (11). Clonal relatedness was established by pulsed-field gel electrophoresis (PFGE) and multilocus sequence typing (MLST) as described previously $(15,22,28)$. Sequence types (STs) differing in one or two of the seven housekeeping genes were considered single-locus variants (SLVs) and double-locus variants, respectively. Clusters of related STs differing in $\leq 2$ loci that were thought to be descendants from a common ancestor were grouped into CCs by using the eBURST software program $(12,13)$ (http://www.mlst .net). Genes coding for virulence factors such as enterococcal surface protein (encoded by esp), hyaluronidase (encoded by $h y l$ ), cytolysin (encoded by $c y l$ ), gelatinase (encoded by gel), and aggregation substance (encoded by agg) and the backbone structure of Tn1546 harbored by vancomycin-resistant $E$. faecium (VRE) were investigated by using PCR $(21,34,38)$.

The isolates studied (35 VRE isolates and 21 vancomycinsusceptible E. faecium [VSE] isolates) corresponded to 49 PFGE types and 41 different STs, including 24 newly identified STs, which clustered into CC5, CC9, CC17, CC22, and CC94 (Fig. 1 and Table 1).

CC17 was identified in 24 isolates (18 VRE isolates and 6 VSE isolates) from hospitals, healthy volunteers, swine, piggeries, and the environment in different regions from 1997 to 2007. In agreement with other studies, the CC17 meroclone consisted of a high diversity of STs (ST16, ST18, ST78, ST80, ST125, ST132, ST280, ST368, ST369, ST390, ST393, ST430, and ST431), particularly enriched by $\operatorname{ST} 18$ (7, 18, 29, 36, 37) (Table 1). All CC17 isolates were resistant to ampicillin and erythromycin, and most of them were associated with resistance to glycopeptides (75\%) and ciprofloxacin (71\%) and a high level of resistance to kanamycin $(58 \%)$ and streptomycin (50\%). esp was detected among isolates from most sources (38\%), while hyl (17\%) was mostly associated with the clinical setting. The detection of CC17 among nonclinical sources might indicate a hospital input of community strains with different genetic contents besides contamination from the hospi- 


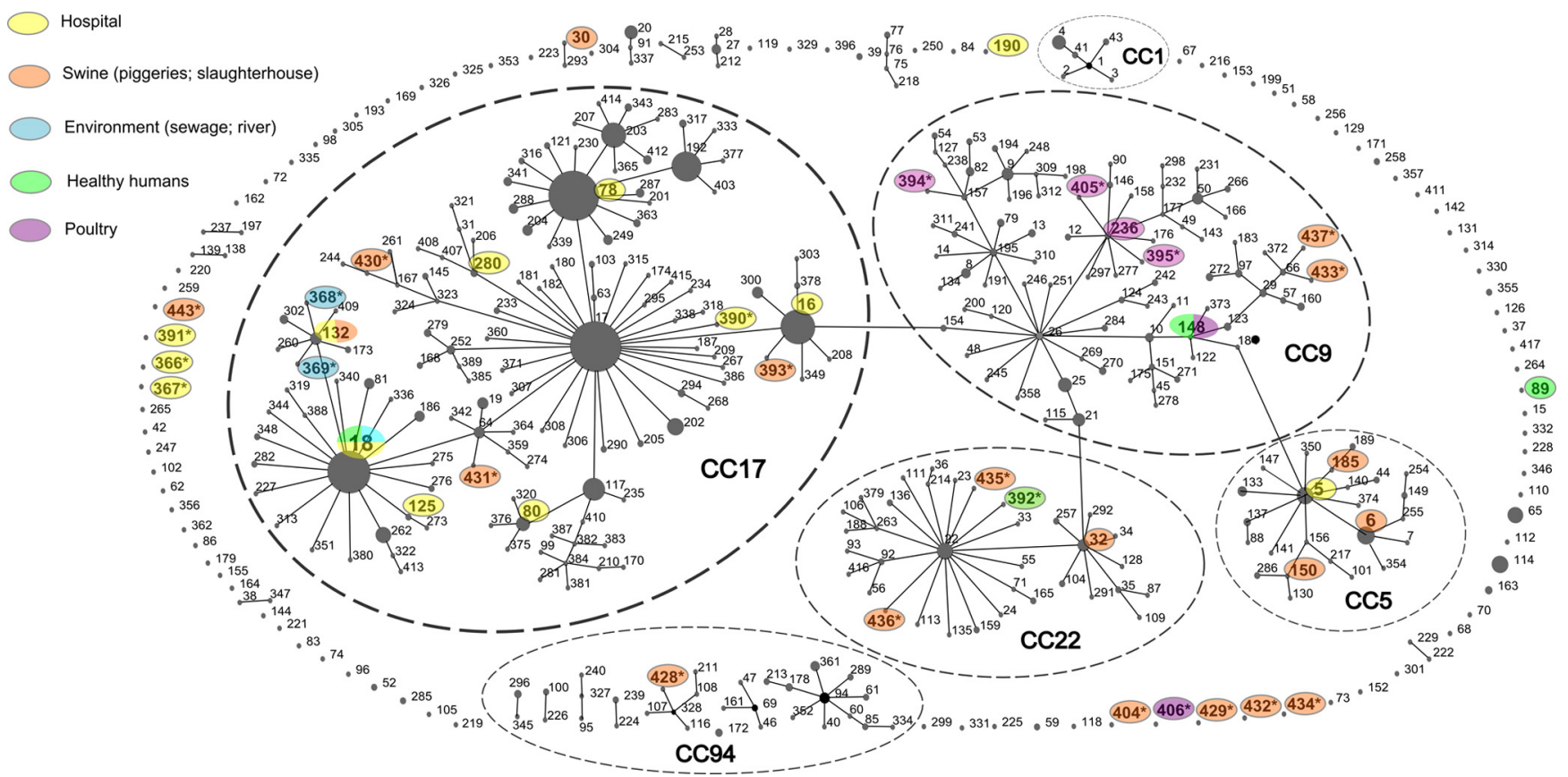

FIG. 1. Clustering of 41 E. faecium STs by use of eBURST. STs are indicated by colored circles, representing 56 isolates from the present study, with 439 MLST profiles representing 1,358 E. faecium isolates from the database (www.mlst.net). Each ST is represented as a node; the relative size of each node is indicative of its prevalence among the isolates, and lines connect SLVs. Colors indicate isolation sources, and CCs are represented by dashed circles (CC1, CC5, CC9, CC17, CC22, and CC94).

tal setting and could explain its high prevalence and global spread $(3,8-10)$.

Clonal dissemination and maintenance of particular resistant E. faecium strains among food animals have previously been documented, but MLST data are absent in almost all of these works $(14,19,26,32,33)$. Although swine and poultry are considered potential reservoirs of pathogenic E. faecium, transmission to humans has been demonstrated in a few cases and is always linked to persons in close contact with farms (4, $19,32,33)$. CC5 comprised isolates of ST5 $(n=1)$, ST6 $(n=$ $1), \operatorname{ST} 150(n=1)$, and ST185 $(n=2)$. Isolates classified as ST5 and ST185, which is an SLV of ST5, were recovered from two hospitals over 2 years (22) and from soil of an extensive piggery, respectively. They were clonally related to the ST6 isolate which represents the VRE strain "A" widespread among swine of four European countries since 1997 (26). CC5 isolates did not contain the tested putative virulence factors and exhibited various resistance phenotypes (Table 1).

CC9 included eight clonally unrelated isolates from community (ST148), swine (ST433 and ST437), and poultry (ST236, ST394, ST395, and ST405), generally resistant to ciprofloxacin $(88 \%)$ and vancomycin (62\%). Most of the CC9 poultry strains were persistently recovered from commercial brands of retail poultry products over the years and corresponded to different ampicillin-resistant VRE or VSE strains (23). All isolates lacked the virulence genes tested. CC9 strains associated with purK6 (ST236 and their SLVs, ST395 and ST405) or purK3 (ST148) have also been detected in chickens from Korea and Spain, respectively, which might mirror particular globally disseminated poultry lineages (http://efaecium.mlst.net/).

CC22 (ST32, ST392, ST435, and ST436) comprised isolates mostly resistant to tetracycline and ciprofloxacin and highly resistant to kanamycin from different piggeries and from human feces. This genogroup was recently associated with isolates from human origin $(5,17)$, with this study representing the first description of isolates among swine.

The remaining STs were recovered from humans and animals in nine different locations and corresponded to ST30, ST89, ST190, ST366, ST367, ST391, ST404, ST406, ST428 (CC94), ST429, ST432, ST434, and ST443, mostly identified as singletons. It is of note that ST366/ST367 and ST391 from hospitals are double-locus variants of STs belonging to CC17 and were resistant to ampicillin and contained esp. Also, ST443 from swine harbored purK1, which is associated with CC17, and contained the esp and hyl genes.

Tn1546 among CC17 isolates was highly diverse, with a number of variants containing ISEf1. Tn1546 types A, D, and S, lacking insertion sequences and associated with animals, were detected in the non-CC17 VRE isolates CC5 and CC9 $(21,38$; this study).

In summary, this is the first study describing the population structure of E. faecium from different origins and locations in Portugal, one of the European nations with the highest VRE rates in the nosocomial setting (see the EARSS Annual Report 2006 [http://www.rivm.nl/earss]) (35). Our results pose new insights into $E$. faecium host specificity, since all the identified clonal complexes comprised isolates from different host origins. The frequent recovery from the community of HiRCC17 and the emergence of CC5 in the hospital setting highlight the role of nonhospital hosts as possible reservoirs of pathogenic enterococci. This community reservoir of multidrug-resistant strains able to cause human infections might contribute to their 


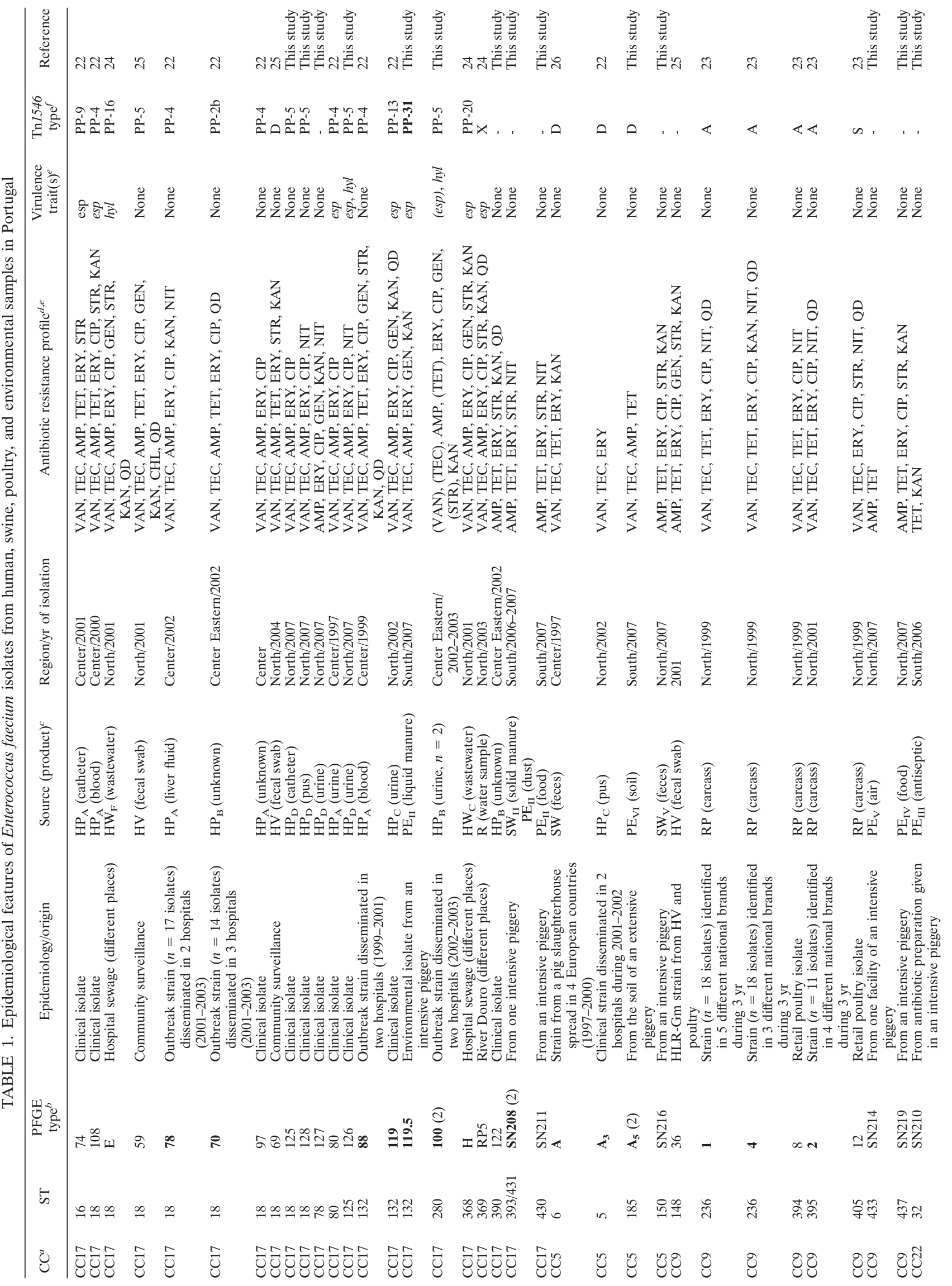




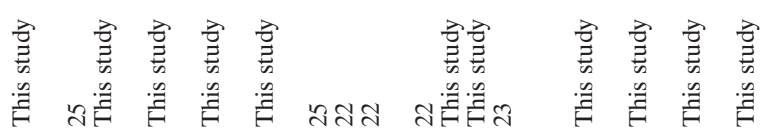
$0 \ll \stackrel{n}{a} \times \frac{n}{2} \ll \ll, \quad, \quad, \quad \varangle$

そัँ

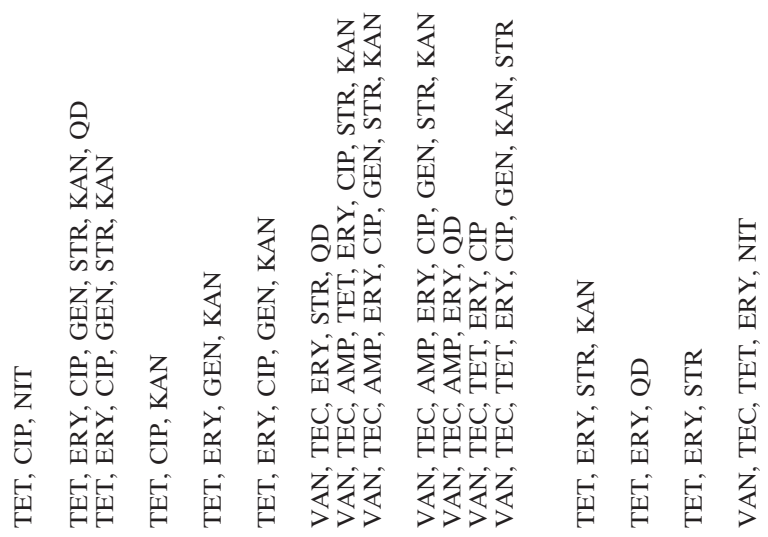

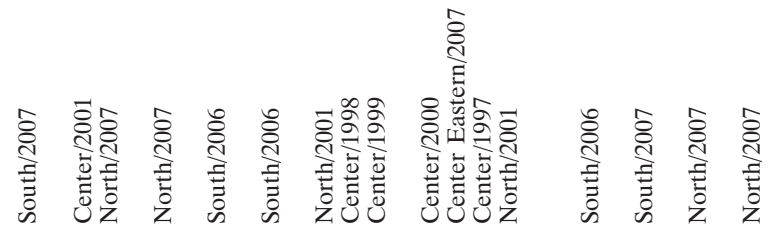

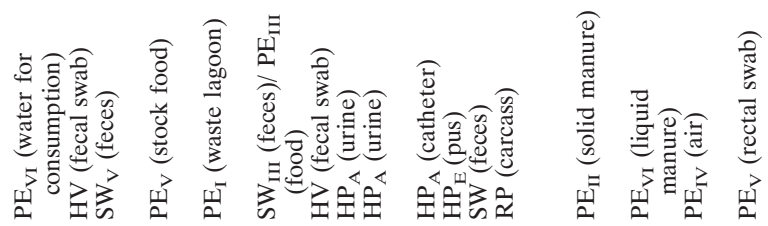

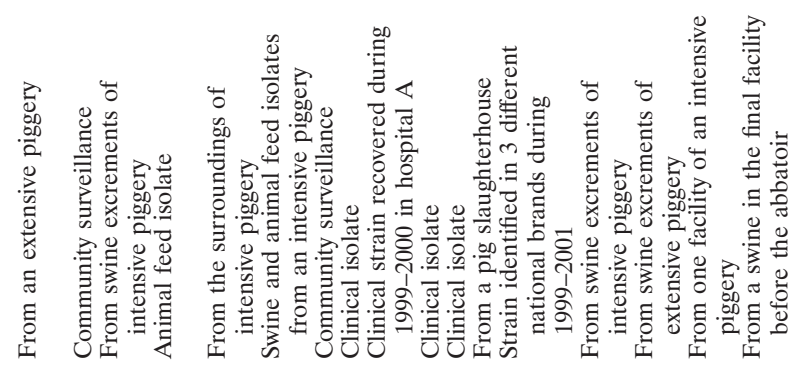

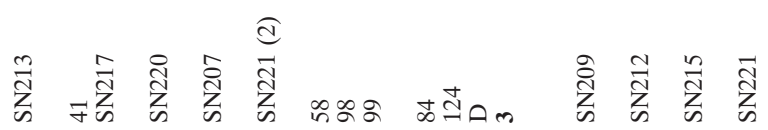

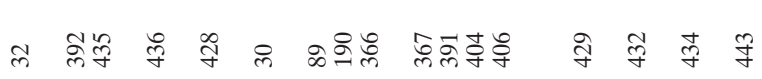

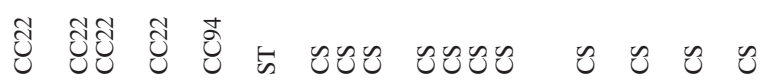

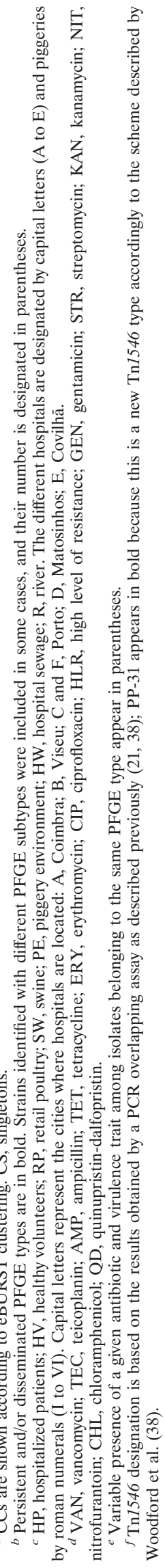


spread in hospitals and counteract any containment measure at the hospital level.

We are indebted to Rob J. Willems (University Utrecht, The Netherlands) for excellent scientific support, assignment of new MLST types, and allocation of new types to clonal complexes (http://www.mlst .net). We are grateful to Jorge M. A. Oliveira (University Porto, Portugal) for his assistance with the eBURST figure.

Ana Freitas was funded by a fellowship from Fundação para a Ciência e Tecnologia (SFRH/BD/24604/2005). This work was funded by grants from Fundação para a Ciência e Tecnologia (POCI/SAL/ 61385/2004 and POCI/AMB/61814/2004) of Portugal, from Ministerio de Educación y Ciencia of Spain, Programa Acciones Integradas Hispano-Portuguesas (H2004-0092), from the Fondo de Investigaciones Sanitarias, Ministerio de Sanidad (PI 06/1141), and from the European Union Sixth Framework Programme (LSHE-CT-2007-037410).

\section{REFERENCES}

1. Aarestrup, F. M., P. Butaye, and W. Witte. 2002. Nonhuman reservoirs of enterococci, p. 55-99. In M. S. Gilmore (ed.), The enterococci: pathogenesis, molecular biology, and antibiotic resistance. American Society for Microbiology, Washington, DC.

2. Abele-Horn, M., U. Vogel, I. Klare, C. Konstabel, R. Trabold, R. Kurihara, W. Witte, W. Kreth, P. G. Schlegel, and H. Claus. 2006. Molecular epidemiology of hospital-acquired vancomycin-resistant enterococci. J. Clin. Microbiol. 44:4009-4013.

3. Biavasco, F., G. Foglia, C. Paoletti, G. Zandri, G. Magi, E. Guaglianone, A. Sundsfjord, C. Pruzzo, G. Donelli, and B. Facinelli. 2007. VanA-type enterococci from humans, animals, and food: species distribution, population structure, Tn1546 typing and location, and virulence determinants. Appl. Environ. Microbiol. 73:3307-3319.

4. Bruinsma, N., R. J. Willems, A. E. van den Bogaard, M. van Santen-Verheuvel, N. London, C. Driessen, and E. E. Stobberingh. 2002. Different levels of genetic homogeneity in vancomycin-resistant and -susceptible Enterococcus faecium isolates from different human and animal sources analyzed by amplified-fragment length polymorphism. Antimicrob. Agents Chemother. 46:2779-2783.

5. Camargo, I. L., M. S. Gilmore, and A. L. Darini. 2006. Multilocus sequence typing and analysis of putative virulence factors in vancomycin-resistant and vancomycin-sensitive Enterococcus faecium isolates from Brazil. Clin. Microbiol. Infect. 12:1123-1130.

6. Clinical Laboratory Standards Institute. 2006. Performance standards for antimicrobial susceptibility testing; 16th informational supplement M100S16, vol. 26. Clinical and Laboratory Standards Institute, Wayne, PA.

7. Coque, T. M., R. J. Willems, J. Fortún, J. Top, S. Diz, E. Loza, R. Cantón, and F. Baquero. 2005. Population structure of Enterococcus faecium causing bacteremia in a Spanish university hospital: setting a scene for a future increase in vancomycin resistance? Antimicrob. Agents Chemother. 49:2693-2700.

8. Damborg, P., A. H. Sørensen, and L. Guardabassi. 2008. Monitoring of antimicrobial resistance in healthy dogs: first report of canine ampicillinresistant Enterococcus faecium clonal complex 17. Vet. Microbiol. 132:190196.

9. Damborg, P., J. Top, A. P. Hendrickx, S. Dawson, R. J. Willems, and L. Guardabassi. 2009. Dogs are a reservoir of ampicillin-resistant Enterococcus faecium lineages associated with human infections. Appl. Environ. Microbiol. 75:2360-2365.

10. De Leener, E., A. Martel, E. M. De Graef, J. Top, P. Butaye, F. Haesebrouck, R. J. Willems, and A. Decostere. 2005. Molecular analysis of human, porcine, and poultry Enterococcus faecium isolates and their erm(B) genes. Appl. Environ. Microbiol. 71:2766-2770.

11. Dutka-Malen, S., S. Evers, and P. Courvalin. 1995. Detection of glycopeptide resistance genotypes and identification to the species level of clinically relevant enterococci by PCR. J. Clin. Microbiol. 33:24-27.

12. Feil, E. J., B. C. Li, D. M. Aanensen, W. P. Hanage, and B. G. Spratt. 2004 eBURST: inferring patterns of evolutionary descent among clusters of related bacterial genotypes from multilocus sequence typing data. J. Bacteriol. 186: $1518-1530$.

13. Feil, E. J., and B. G. Spratt. 2001. Recombination and the population structures of bacterial pathogens. Annu. Rev. Microbiol. 55:561-590.

14. Heuer, O. E., K. Pedersen, L. B. Jensen, M. Madsen, and J. E. Olsen. 2002 Persistence of vancomycin-resistant enterococci (VRE) in broiler houses after the avoparcin ban. Microb. Drug Resist. 8:355-361.

15. Homan, W. L., D. Tribe, S. Poznanski, M. Li, G. Hogg, E. Spalburg, J. D. van Embden, and R. J. Willems. 2002. Multilocus sequence typing scheme for Enterococcus faecium. J. Clin. Microbiol. 40:1963-1971.

16. Klare, I., C. Konstabel, S. Mueller-Bertling, G. Werner, B. Strommenger, C.
Kettlitz, S. Borgmann, B. Schulte, D. Jonas, A. Serr, A. M. Fahr, U. Eigner, and W. Witte. 2005. Spread of ampicillin/vancomycin-resistant Enterococcus faecium of the epidemic-virulent clonal complex-17 carrying the genes esp and hyl in German hospitals. Eur. J. Clin. Microbiol. Infect. Dis. 24:815-825.

17. Leavis, H. L., M. J. Bonten, and R. J. Willems. 2006. Identification of high-risk enterococcal clonal complexes: global dispersion and antibiotic resistance. Curr. Opin. Microbiol. 9:454-460.

18. Lester, C. H., D. Sandvang, S. S. Olsen, H. C. Schønheyder, J. O. Jarløv, J. Bangsborg, D. S. Hansen, T. G. Jensen, N. Frimodt-Møller, and A. M. Hammerum. 2008. Emergence of ampicillin-resistant Enterococcus faecium in Danish hospitals. J. Antimicrob. Chemother. 62:1203-1206.

19. Lu, H. Z., X. H. Weng, H. Li, Y. K. Yin, M. Y. Pang, and Y. W. Tang. 2002. Enterococcus faecium-related outbreak with molecular evidence of transmission from pigs to humans. J. Clin. Microbiol. 40:913-917.

20. Murray, B. E. 2000. Vancomycin resistant enterococcal infections. N. Engl. J. Med. 342:710-721.

21. Novais, C., A. R. Freitas, J. C. Sousa, F. Baquero, T. M. Coque, and L. Peixe. 2008. Diversity of Tn1546 and its role in the dissemination of vancomycinresistant enterococci in Portugal. Antimicrob. Agents Chemother. 52:10011008 .

22. Novais, C., J. C. Sousa, T. M. Coque, and L. Peixe. 2005. Molecular characterization of glycopeptide-resistant Enterococcus faecium isolates from Portuguese hospitals. Antimicrob. Agents Chemother. 49:3073-3079.

23. Novais, C., T. M. Coque, M. J. Costa, J. C. Sousa, F. Baquero, and L. Peixe. 2005. High occurrence and persistence of antibiotic-resistant enterococci in poultry food samples in Portugal. J. Antimicrob. Chemother. 56:1139-1143.

24. Novais, C., T. M. Coque, H. Ferreira, J. C. Sousa, and L. Peixe. 2005. Environmental contamination with vancomycin-resistant enterococci from hospital sewage in Portugal. Appl. Environ. Microbiol. 71:3364-3368.

25. Novais, C., T. M. Coque, J. C. Sousa, and L. V. Peixe. 2006. Antimicrobial resistance among faecal enterococci from healthy individuals in Portugal. Clin. Microbiol. Infect. 12:1131-1134.

26. Novais, C., T. M. Coque, P. Boerlin, I. Herrero, M. A. Moreno, L. Dominguez, and L. Peixe. 2005. Vancomycin-resistant Enterococcus faecium clone in swine, Europe. Emerg. Infect. Dis. 11:1985-1987.

27. Stampone, L., M. Del Grosso, D. Boccia, and A. Pantosti. 2005. Clonal spread of a vancomycin-resistant Enterococcus faecium strain among bloodstream-infecting isolates in Italy. J. Clin. Microbiol. 43:1575-1580.

28. Tenover, F. C., R. D. Arbeit, R. V. Goering, P. A. Mickelsen, B. E. Murray, D. H. Persing, and B. Swaminathan. 1995. Interpreting chromosomal DNA restriction patterns produced by pulsed-field gel electrophoresis: criteria for bacterial strain typing. J. Clin. Microbiol. 33:2233-2239.

29. Top, J., R. Willems, S. Van der Velden, M. Asbroek, and M. Bonten. 2008 Emergence of clonal complex 17 Enterococcus faecium in The Netherlands. J. Clin. Microbiol. 46:214-219.

30. Top, J., R. Willems, and M. Bonten. 2008. Emergence of CC17 Enterococcus faecium: from commensal to hospital-adapted pathogen. FEMS Immunol. Med. Microbiol. 52:297-308.

31. Reference deleted.

32. van den Bogaard, A. E., R. Willems, N. London, J. Top, and E. E. Stobberingh. 2002. Antibiotic resistance of faecal enterococci in poultry, poultry farmers and poultry slaughterers. J. Antimicrob. Chemother. 49:497-505.

33. van den Braak, N., A. van Belkum, M. van Keulen, J. Vliegenthart, H. A. Verbrugh, and H. P. Endtz. 1998. Molecular characterization of vancomycinresistant enterococci from hospitalized patients and poultry products in The Netherlands. J. Clin. Microbiol. 36:1927-1932.

34. Vankerckhoven, V., T. van Autgaerden, C. Vael, C. Lammens, S. Chapelle, R. Rossi, D. Jabes, and H. Goossens. 2004. Development of a multiplex PCR for the detection of asal, gelE, cylA, esp, and hyl genes in enterococci and survey for virulence determinants among European hospital isolates of Enterococcus faecium. J. Clin. Microbiol. 42:4473-4479.

35. Werner, G., T. M. Coque, A. M. Hammerum, R. Hope, W. Hryniewicz, A. Johnson, I. Klare, K. G. Kristinsson, R. Leclercq, C. H. Lester, M. Lillie, C. Novais, B. Olsson-Liljequist, L. V. Peixe, E. Sadowy, G. S. Simonsen, J. Top, J. Vuopio-Varkila, R. J. Willems, W. Witte, and N. Woodford. 2008. Emergence and spread of vancomycin resistance among enterococci in Europe. Euro Surveill. 13:pii $=19046$.

36. Werner, G., I. Klare, C. Fleige, and W. Witte. 2008. Increasing rates of vancomycin resistance among Enterococcus faecium isolated from German hospitals between 2004 and 2006 are due to wide clonal dissemination of vancomycin-resistant enterococci and horizontal spread of $\operatorname{van} A$ clusters. Int. J. Med. Microbiol. 298:515-527.

37. Willems, R. J. L., J. Top, M. van Santen, D. A. Robinson, T. M. Coque, F. Baquero, H. Grundmann, and M. J. Bonten. 2005. Global spread of vancomycin-resistant Enterococcus faecium from distinct nosocomial genetic complex. Emerg. Infect. Dis. 11:821828.

38. Woodford, N., A. A. Adebiyi, M. I. Palepou, and B. Cookson. 1998. Diversity of VanA glycopeptide resistance elements in enterococci from humans and nonhuman sources. Antimicrob. Agents Chemother. 42:502-508. 